\title{
A new bound on the capacity of the binary deletion channel with high deletion probabilities
}

\author{
Marco Dalai, \\ Department of Information Engineering \\ University of Brescia, Italy \\ Email: marco.dalai@ing.unibs.it
}

\begin{abstract}
Let $C(d)$ be the capacity of the binary deletion channel with deletion probability $d$. It was proved by Drinea and Mitzenmacher that, for all $d, C(d) /(1-d) \geq 0.1185$. Fertonani and Duman recently showed that $\limsup _{d \rightarrow 1} \bar{C}(d) /(1-d) \leq 0.49$. In this paper, it is proved that $\lim _{d \rightarrow 1} C(d) /(1-d)$ exists and is equal to $\inf _{d} C(d) /(1-d)$. This result suggests the conjecture that the curve $C(d)$ my be convex in the interval $d \in[0,1]$. Furthermore, using currently known bounds for $C(d)$, it leads to the upper bound $\lim _{d \rightarrow 1} C(d) /(1-d) \leq 0.4143$.
\end{abstract}

\section{INTRODUCTION}

A binary deletion channel $W^{d}$ is defined as a binary channel that drops bits of the input sequence independently with probability $d$. Those bits that are not dropped simply pass through the channel unaltered. While simple to describe, the deletion channel proves to be very difficult to analyze. Dobrushin ([1]) showed that for such a channel it is possible to define a capacity $C(d)$ and that a Shannon like theorem applies to this channel. However, no closed formula expression is known up to now for the capacity $C(d)$, and only upper and lower bounds are currently available (see [2], [3], [4], [5], [6]).

For small values of $d$, it was recently independently proved in [4] and [5] that $C(d) \approx 1-H(d)$, where $H(d)$ is the binary entropy function. For values of $d$ close to 1 , it is known (see [7], [6]) that $C(d)$ satisfies

$$
0.1185 \leq \liminf _{d \rightarrow 1} \frac{C(d)}{1-d} \leq \limsup _{d \rightarrow 1} \frac{C(d)}{1-d} \leq 0.49
$$

As far as the author knows, there is no result in the literature on the existence of $\lim _{d \rightarrow 1} C(d) /(1-d)$. In this paper, it is proved that the limit exists and, in particular, that

$$
\lim _{d \rightarrow 1} \frac{C(d)}{(1-d)}=\inf _{d} \frac{C(d)}{(1-d)} .
$$

The best currently known upper bound for $C(d)$, when used in the right hand side of (2), leads to the upper bound

$$
\lim _{d \rightarrow 1} \frac{C(d)}{(1-d)} \leq 0.4143
$$

which improves the best previously known bound of equation (1). Furthermore, equation (2) suggests the conjecture that $C(d)$ may be a convex function of $d$. Indeed, as discussed in Section IV below, experimental evidence (see Figure 1) suggests the convexity of $C(d)$ for values of $d$ sufficiently smaller than 1 , while it is not easy to exclude that the function may be concave near $d=1$. Equation (2) is only a necessary condition ${ }^{1}$ for the convexity of $C(d)$ near $d=1$. It is, however, sufficient to conclude that $C(d)$ is not strictly concave in any neighborhood of $d=1$. Thus, either $C(d)$ exhibit a pathological behavior near $d=1$, or it is convex in a sufficiently small neighborhood of $d=1$. A proof of the convexity of $C(d)$ would of course imply equation (2) and thus equation (3).

The main idea used in this paper is the intuitive fact that, for a large enough number of input bits $n$, the deletion channel $W^{d}$ is fairly well approximated by a channel which drops exactly $[d n]$ bits selected uniformly at random. In particular, we show that a channel $W_{n, k}$ with $n$-bits input and $k$-bits output, selected uniformly within the $k$-bits subsequences of the input, has a capacity that is close to $C(1-k / n)$ for large enough $n$. Using this result, we build upon the work in [6] to prove (2).

\section{Definition And Regularity of $C(d)$}

For any $i$ and $j$, let $X_{i}^{j}=\left(X_{i}, X_{i+1}, \ldots, X_{j}\right)$ and, similarly $Y_{i}^{j}=\left(Y_{i}, Y_{i+1} \ldots, Y_{j}\right)$. Let $W_{n}^{d}$ be a channel with an $n$-bit string input whose output is obtained by dropping the bits of the input independently with probability $d$. Let then

$$
C_{n}(d)=\frac{1}{n} \max _{p_{X_{1}^{n}}} I\left(X_{1}^{n} ; W_{n}^{d}\left(X_{1}^{n}\right)\right) .
$$

It was proved by Dobrushin [1] that a transmission capacity $C(d)$ can be consistently defined for the deletion channel $W^{d}$ and that it holds

$$
C(d)=\lim _{n \rightarrow \infty} C_{n}(d) .
$$

Figure 1 shows the graph of the $C_{n}(d)$ functions for $n=$ $1, \ldots, 17$. The main objective of this section is to study the convergence of the $C_{n}(d)$ functions to deduce a regularity result for $C(d)$.

The following lemma gives a quantitative bound on the rate of convergence in (5).

Lemma 1: (see also [1], [4], [6]) For every $d \in[0,1]$ and $n \geq 1$

$$
C_{n}(d)-\frac{\log (n+1)}{n} \leq C(d) \leq C_{n}(d) .
$$

\footnotetext{
${ }^{1}$ It is not difficult to construct examples of "pathological" functions $f(d)$ that satisfy equation (2), when used in place of $C(d)$, but are not convex in any neighborhood of $d=1$.
} 


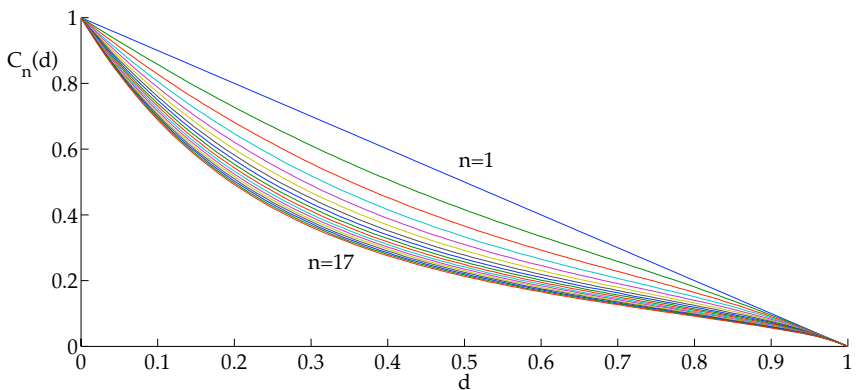

Fig. 1. Plot of the $C_{n}(d)$ functions for $n=1 \ldots 17$ obtained by numerical evaluations in [6].

Proof: As observed in [4], $n C_{n}(d)$ is a subadditive function of $n$. In fact, for an input $X_{1}^{n+m}$, let $\tilde{Y}_{(0)}=W_{n}^{d}\left(X_{1}^{n}\right)$ and $\tilde{Y}_{(1)}=W_{m}^{d}\left(X_{n+1}^{n+m}\right)$. Note that $Y=W_{n+m}^{d}\left(X_{1}^{n+m}\right)$ can be obtained as a concatenation of the strings $\tilde{Y}_{(0)}$ and $\tilde{Y}_{(1)}$. Thus, $X_{1}^{n+m} \rightarrow\left(\tilde{Y}_{(0)}, \tilde{Y}_{(1)}\right) \rightarrow Y$ is a Markov chain. Hence,

$$
\begin{aligned}
(n+m) C_{n+m}(d) & =\max _{p_{X_{1}^{n+m}}} I\left(X_{1}^{n+m} ; Y\right) \\
& \leq \max _{p_{X_{1}^{n+m}}} I\left(X_{1}^{n+m} ;\left(\tilde{Y}_{(0)}, \tilde{Y}_{(1)}\right)\right) \\
& \leq n C_{n}(d)+m C_{m}(d) .
\end{aligned}
$$

This implies by Fekete's lemma (see [8, Prob. 98]) that the limit $C(d)=\lim _{n \rightarrow \infty} C_{n}(d)$ exists and it satisfies $C(d)=$ $\inf _{n \geq 1} C_{n}(d)$. This proves the right hand side inequality.

Take now an integer $h>1$ and consider, for an input $X_{1}^{h n}$, the output $Y=W_{h n}^{d}\left(X_{1}^{h n}\right)$ as the concatenation of the $h$ outputs $\tilde{Y}_{(i)}=W_{n}^{d}\left(X_{n i+1}^{n i+n}\right), i=0, \ldots, h-1$. Let for convenience $\tilde{Y}_{(0)}^{(h-1)}=\left(\tilde{Y}_{(0)}, \tilde{Y}_{(1)}, \ldots, \tilde{Y}_{(h-1)}\right)$. It is clear that $X_{1}^{h n} \rightarrow \tilde{Y}_{(0)}^{(h-1)} \rightarrow Y$ is a Markov Chain. Let $L_{i}$ be the length of $\tilde{Y}_{(i)}$. We thus have

$$
\begin{aligned}
h n C_{h n}(d) & =\max _{p_{X_{1}^{h n}}} I\left(X_{1}^{h n} ; Y\right) \\
& =\max _{p_{X_{1}^{h n}}}\left[I\left(X_{1}^{h n} ; \tilde{Y}_{(0)}^{(h-1)}\right)-I\left(X_{1}^{h n} ; \tilde{Y}_{(0)}^{(h-1)} \mid Y\right)\right] \\
& \geq \max _{p_{X_{1}^{h n}}}\left[I\left(X_{1}^{h n} ; \tilde{Y}_{(0)}^{(h-1)}\right)-H\left(\tilde{Y}_{(0)}^{(h-1)} \mid Y\right)\right] \\
& =\max _{p_{X_{1}^{h n}}}\left[I\left(X_{1}^{h n} ; \tilde{Y}_{(0)}^{(h-1)}\right)-H\left(L_{0}^{h-1} \mid Y\right)\right] \\
& \geq \max _{p_{X_{1}^{h n}}} I\left(X_{1}^{h n} ; \tilde{Y}_{(0)}^{(h-1)}\right)-(h-1) \log (n+1) \\
& =h_{n} C_{n}(d)-(h-1) \log (n+1) .
\end{aligned}
$$

Hence

$$
\begin{aligned}
C(d) & =\lim _{h \rightarrow \infty} C_{h n}(d) \\
& \geq \lim _{h \rightarrow \infty}\left[C_{n}(d)-\frac{h-1}{h} \frac{\log (n+1)}{n}\right] \\
& =C_{n}(d)-\frac{\log (n+1)}{n} .
\end{aligned}
$$

See [6, eq. (39)] for tighter, though more complicated, bound.

As a consequence of Lemma 1 we have the following regularity result for $C(d)$.

Lemma 2: The function $C(d)$ is uniformly continuous in $[0,1]$. Thus, for every $\beta>0$ there is a $\alpha=\alpha(\beta)$ such that $\left|d_{1}-d_{2}\right|<\alpha \Rightarrow\left|C\left(d_{1}\right)-C\left(d_{2}\right)\right|<\beta$.

Proof: As shown in Lemma 1, the functions $C_{n}(d)$ tend to $C(d)$ uniformly in $d$. Hence, if proved that the $C_{n}(d)$ are continuous in $d$, so is their limit $C(d)$. Since the domain of $C(d)$ is compact, by the Heine-Cantor theorem $C(d)$ is also uniformly continuous. That the $C_{n}(d)$ functions are continuous is really intuitive; the shortest formal proof that we were able to provide goes as follows. The entries of the transition matrix of the channel $W_{n}^{d}$ are polynomials in $d$ and thus the mutual information $I\left(X_{1}^{n} ; W_{n}^{d}\left(X_{1}^{n}\right)\right)$ is a continuous function of $d$ and of the input distribution $p_{X_{1}^{n}}$. Hence, by moving $d$ continuously from 0 to 1 one expects the capacity to change continuously from 1 to 0 . A formal proof, however, seems to require using the compactness of the sets of distributions $p_{X_{1}^{n}}$. Assume that $C_{n}(d)$ is not continuous in $d=\bar{d}$ and let $\bar{p}$ be the input distribution that attains the value $C_{n}(\bar{d})$. Then there exists an $\varepsilon>0$ such that $\left|C_{n}(\bar{d})-C_{n}\left(d_{k}\right)\right|>\varepsilon$ for a sequence $d_{k}$ converging to $\bar{d}$. Consider the distributions $p_{k}$ that attain $C_{n}\left(d_{k}\right)$. Since the set of the $p_{X_{1}^{n}}$ is bounded and closed, there exists a subsequence of the $p_{k}$ that converges to a distribution $p^{\prime}$. By continuity of the mutual information the $C_{n}\left(d_{k}\right)$ values tend to the mutual information $I^{\prime}$ attained by $p^{\prime}$ in $d=\bar{d}$. But, by definition of $C_{n}(\bar{d})$, we clearly have that $I^{\prime} \leq C_{n}(\bar{d})$ and thus $C_{n}\left(d_{k}\right) \leq C_{n}(\bar{d})-\varepsilon$ for $k$ large enough. But then the mutual information attained by $\bar{p}$ in $d_{k}$ tends to $C_{n}(\bar{d}) \geq C_{n}\left(d_{k}\right)+\varepsilon$ for large enough $k$, which is absurd by definition of $C_{n}\left(d_{k}\right)$.

\section{EXACT DELETION CHANNEL}

Let now $W_{n, k}, k \leq n$, be a channel with $n$-bits input whose output is uniformly chosen within the $\left(\begin{array}{l}n \\ k\end{array}\right) k$-bits subsequences of the input. This channel was efficiently used as an auxiliary channel in [5], [6]. Let then

$$
C_{n, k}=\frac{1}{n} \max _{p_{X_{1}^{n}}} I\left(X_{1}^{n} ; W_{n, k}\left(X_{1}^{n}\right)\right) .
$$

The following obvious result will be used later.

Lemma 3: For every random $X_{1}^{n}$, if $k_{1} \geq k_{2}$ then

$$
I\left(X_{1}^{n} ; W_{n, k_{1}}\left(X_{1}^{n}\right)\right) \geq I\left(X_{1}^{n} ; W_{n, k_{2}}\left(X_{1}^{n}\right)\right) .
$$

Proof: Simply note that the $W_{n, k_{2}}$ channel can be obtained as a cascade of $W_{n, k_{1}}$ and $W_{k_{1}, k_{2}}$. Thus, $X_{1}^{n} \rightarrow$ $W_{n, k_{1}}\left(X_{1}^{n}\right) \rightarrow W_{n, k_{2}}\left(X_{1}^{n}\right)$ is a Markov chain and the lemma follows from the data processing inequality.

The following lemma bounds the capacity of the $W_{n}^{d}$ channel in terms of the capacity of certain exact deletion channels.

Lemma 4: For every $\varepsilon>0, d \in[\varepsilon, 1-\varepsilon]$, and $n \geq 1$

$C_{n,\lceil(1-d-\varepsilon) n\rceil}-2 e^{-2 \varepsilon^{2} n} \leq C_{n}(d) \leq C_{n,\lfloor(1-d+\varepsilon) n\rfloor}+2 e^{-2 \varepsilon^{2} n}$ 
Proof: We first prove the right hand side inequality. For an input $X_{1}^{n}$, let $Y=W_{n}^{d}\left(X_{1}^{n}\right)$ and let $L=|Y|$ be the length of $Y$. First note that $X_{1}^{n} \rightarrow Y \rightarrow L$ is a Markov chain. So, by applying the chain rule to $I\left(X_{1}^{n} ; Y, L\right)$, considered that $I\left(X_{1}^{n} ; L\right)=0$ since $L$ is independent from $X_{1}^{n}$, it is easily seen that $I\left(X_{1}^{n} ; Y\right)=I\left(X_{1}^{n} ; Y \mid L\right)$. Define $T=\left\{j:\left|\frac{j}{n}-(1-d)\right| \leq \varepsilon\right\}$, that is $j \in T$ if and only if $\lceil(1-d-\varepsilon) n\rceil \leq j \leq\lfloor(1-d+\varepsilon) n\rfloor$. Let now $X_{1}^{n}$ be distributed according to the optimal distribution for the $W_{n}^{d}$ channel. Then we have

$$
\begin{aligned}
n C_{n}(d) & =I\left(X_{1}^{n} ; Y \mid L\right) \\
& =\sum_{j=0}^{n} p_{L}(j) I\left(X_{1}^{n} ; Y \mid L=j\right) \\
& =\sum_{j \in T} p_{L}(j) I\left(X_{1}^{n} ; Y \mid L=j\right) \\
& +\sum_{j \in \bar{T}} p_{L}(j) I\left(X_{1}^{n} ; Y \mid L=j\right) \\
& \stackrel{(a)}{\leq} \sum_{j \in T} p_{L}(j) I\left(X_{1}^{n} ; Y \mid L=\lfloor(1-d+\varepsilon) n\rfloor\right) \\
& +\sum_{j \in \bar{T}} p_{L}(j) n \\
& \leq n C_{n,\lfloor(1-d+\varepsilon) n\rfloor} \sum_{j \in T} p_{L}(j)+n \sum_{j \in \bar{T}} p_{L}(j) \\
& \stackrel{(b)}{\leq} \quad n C_{n, L(1-d+\varepsilon) n\rfloor}+2 n e^{-2 \varepsilon^{2} n},
\end{aligned}
$$

where $(a)$ follows from Lemma 3 and the definition of $T$ and (b) follows from the Chernoff bound. Dividing by $n$ we get the desired inequality.

As for the left hand side inequality, let now $X_{1}^{n}$ be distributed according to the optimal distribution for the $W_{n,\lceil(1-d-\varepsilon) n\rceil}$ channel. Then we have

$$
\begin{aligned}
n C_{n}(d) & \geq I\left(X_{1}^{n} ; Y \mid L\right) \\
& =\sum_{j=0}^{n} p_{L}(j) I\left(X_{1}^{n} ; Y \mid L=j\right) \\
& =\sum_{j \in T} p_{L}(j) I\left(X_{1}^{n} ; Y \mid L=j\right) \\
& +\sum_{j \in \bar{T}} p_{L}(j) I\left(X_{1}^{n} ; Y \mid L=j\right) \\
& \stackrel{(a)}{\geq} \sum_{j \in T} p_{L}(j) I\left(X_{1}^{n} ; Y \mid L=\lceil(1-d-\varepsilon) n\rceil\right) \\
& =n C_{n,\lceil(1-d-\varepsilon) n\rceil} \sum_{j \in T} p_{L}(j) \\
& \stackrel{(b)}{\geq}{ } C_{n,\lceil(1-d+\varepsilon) n\rceil}\left(1-2 e^{-2 \varepsilon^{2} n}\right) \\
& \stackrel{(c)}{\geq} n C_{n,\lceil(1-d+\varepsilon) n\rceil}-2 n e^{-2 \varepsilon^{2} n},
\end{aligned}
$$

where $(a)$ follows again from Lemma 3, (b) follows from the Chernoff bound, and $(c)$ follows from the obvious fact that $C_{n,[(1-d+\varepsilon) n\rceil} \leq 1$. Dividing by $n$ the desired result is obtained.

The following lemma bounds the capacity of the exact deletion channel $W_{n, k}$ in terms of $C(d)$ for appropriate values of $d$.

Lemma 5: For every $\varepsilon>0$ and integers $n$ and $k$

$$
\begin{aligned}
C(1-k / n+\varepsilon)-2 e^{-2 \varepsilon^{2} n} & \leq C_{n, k} \leq C(1-k / n-\varepsilon) \\
& +2 e^{-2 \varepsilon^{2} n}+\frac{\log (n+1)}{n}
\end{aligned}
$$

Proof: Take $d=1-k / n-\varepsilon$ in Lemma 4 to obtain $C_{n, k} \leq C_{n}(1-k / n-\varepsilon)+2 e^{-2 \varepsilon^{2} n} \leq C(1-k / n-\varepsilon)+$ $2 e^{-2 \varepsilon^{2} n}+\log (n+1) / n$, by virtue of Lemma 1 . Then take $d=1-k / n+\varepsilon$ in Lemma 4 to obtain $C_{n, k} \geq C_{n}(1-k / n+$ $\varepsilon)-2 e^{-2 \varepsilon^{2} n} \geq C(1-k / n+\varepsilon)-2 e^{-2 \varepsilon^{2} n}$.

Lemma 6: For every $\beta>0$, there is an $\bar{n}=\bar{n}(\beta)$ such that

$$
\left|C_{n, k}-C(1-k / n)\right|<\beta \quad \forall n \geq \bar{n}, k=1, \ldots, n .
$$

Proof: First note that, for $\varepsilon>0, C(1-k / n+\varepsilon) \leq$ $C(1-k / n) \leq C(1-k / n-\varepsilon)$. Hence, $C(1-k / n)$ satisfies the two inequalities satisfied by $C_{n, k}$ in equation (10). So, $\left|C_{n, k}-C(1-k / n)\right|$ is bounded by the difference between the right hand side and the left hand side of equation (10), that is

$$
\begin{array}{r}
\left|C_{n, k}-C(1-k / n)\right| \leq C(1-k / n-\varepsilon)-C(1-k / n+\varepsilon) \\
+4 e^{-2 \varepsilon^{2} n}+\frac{\log (n+1)}{n} .
\end{array}
$$

With the notation of Lemma 2, take $\varepsilon<\alpha(\beta / 2) / 2$ so that $C(1-k / n-\varepsilon)-C(1-k / n+\varepsilon)<\beta / 2$. Once $\varepsilon$ is fixed, choose $\bar{n}$ such that $4 e^{-2 \varepsilon^{2} \bar{n}}+\frac{\log (\bar{n}+1)}{\bar{n}}<\beta / 2$ to complete the proof. Note that $\bar{n}$ is a function of $\beta$ only and that the result holds for every $k \leq n$.

We can now state the first result of this paper.

Theorem 1: Let $k_{n}$ be an integer valued sequence such that $k_{n} / n$ tends to $1-d$ as $n$ goes to infinity. Then

$$
\lim _{n \rightarrow \infty} C_{n, k_{n}}=C(d) \text {. }
$$

Proof: It follows easily from Lemma 6 by continuity of $C(d)$.

\section{BEHAVIOR NEAR $d=1$}

In this Section, we finally focus on the behavior of the function $C(d)$ for values of $d$ close to 1 . It is interesting to observe in Figure 1 that, from experimental evidence, the $C_{n}(d)$ functions seem to be convex in a progressively expanding region of $d$ values. On the one hand, it is tempting to conjecture that the limit $C(d)$ is convex in the whole interval $d \in[0,1]$. On the other hand, near $d=1$, all the $C_{n}(d)$ curves appear to change concavity and go to zero asymptotically as $(1-d)$. Indeed, we have the following result.

Lemma 7: For every $n$,

$$
\lim _{d \rightarrow 1} \frac{C_{n}(d)}{(1-d)}=1
$$


Proof: It is easily shown that for every $n$ and $d$

$$
\left(1-d^{n}\right) / n \leq C_{n}(d)<(1-d) .
$$

The right hand side inequality follows from the fact that the capacity of $W_{n}^{d}$ is obviously smaller than the capacity of a binary erasure channel with erasure probability $d$. To prove the left hand side inequality consider using as input to the channel $W_{n}^{d}$ only the sequence composed of $n$ zeros and that composed of $n$ ones. Then the $n$ uses of $W_{n}^{d}$ correspond to one use of an erasure channel with erasure probability $d^{n}$. This proves equation (15). Dividing by $(1-d)$ and taking the limit $d \rightarrow 1$ gives the required result.

Lemma 7 ensures that, for fixed $n, C_{n}(d)$ is not convex in a neighborhood of $d=1$. Note further that

$$
\lim _{d \rightarrow 1} \frac{C_{n}(d)}{(1-d)}=\sup _{d \in(0,1)} \frac{C_{n}(d)}{(1-d)}=1
$$

Hence, it is natural to believe that $C_{n}(d)$ is actually concave in a neighborhood of $d=1$, even if Lemma 7 is not sufficient to prove this. However, in the limit $n \rightarrow \infty$, it is known (see [7], [6]) that $C(d)$ satisfies

$$
0.1185 \leq \liminf _{d \rightarrow 1} \frac{C(d)}{1-d} \leq \limsup _{d \rightarrow 1} \frac{C(d)}{1-d} \leq 0.49
$$

Hence, Lemma 7 does not hold with $C(d)$ in place of $C_{n}(d)$ and it is still legitimate to conjecture that $C(d)$ may be convex in $[0,1]$. The next step is thus to ask if $C_{n}(d) /(1-d)$ has a limit as $d \rightarrow 1$ and, if so, if this limit is reached from above as would be implied by convexity of $C(d)$. The remaining part of this section tries to answer this question.

In order to understand the behavior of $C(d)$ near $d=1$, the following result from [6] is fundamental.

Lemma 8 (Fertonani and Duman, [6, eq. (32)]): For every $n, k$

$$
\limsup _{d \rightarrow 1} \frac{C(d)}{1-d} \leq \frac{n C_{n, k}+1}{k+1} .
$$

Remark 1: In [6] the authors state that, for every $n$ and $k, \lim _{d \rightarrow 1} \frac{C(d)}{1-d} \leq \frac{n C_{n, k}+1}{k+1}$. However, we are not aware of a previous formal proof that $\lim _{d \rightarrow 1} \frac{C(d)}{1-d}$ exists. This fact is proved in the following theorem.

Theorem 2: It holds that

$$
\lim _{d \rightarrow 1} \frac{C(d)}{(1-d)}=\inf _{d \in(0,1)} \frac{C(d)}{1-d} .
$$

Proof: For every $d^{\prime} \in(0,1)$, let $k_{n}$ be a sequence such that $k_{n} / n$ tends to $1-d^{\prime}$. Then, from Theorem 1 , the right hand side of (18), with $k_{n}$ in place of $k$, tends to $C\left(d^{\prime}\right) /\left(1-d^{\prime}\right)$. Since $d^{\prime}$ is arbitrary, Lemma 8 implies that $\lim \sup _{d \rightarrow 1} C(d) /(1-d) \leq \inf _{d^{\prime} \in(0,1)} \frac{C\left(d^{\prime}\right)}{1-d^{\prime}}$. However, it is obvious that $\liminf _{d \rightarrow 1} C(d) /(1-d) \geq \inf _{d^{\prime} \in(0,1)} \frac{C\left(d^{\prime}\right)}{1-d^{\prime}}$. Thus $\lim _{d \rightarrow 1} C(d) /(1-d)$ exists and is equal to $\inf _{d^{\prime} \in(0,1)} \frac{C\left(d^{\prime}\right)}{1-d^{\prime}}$

A direct consequence of Theorem 2 is the following improved bound on $C(d)$.

\section{Corollary 1:}

$$
\lim _{d \rightarrow 1} \frac{C(d)}{(1-d)} \leq 0.4143
$$

Proof: As far as the author knows, the best known numerical bound obtained for $\inf _{d} C(d) /(1-d)$ is 0.4143 obtained using the bound $C(0.65) \leq C_{17}(0.65)=0.145$, numerically evaluated in [6].

The usefulness of Theorem 2 is that it allows to deduce provable bounds for $\lim _{d \rightarrow 1} \frac{C(d)}{(1-d)}$ from bounds on $C(d)$ even with $d$ much smaller than 1 . It is interesting to note, in fact, that different techniques seem to be effective in bounding $C(d)$ in different regions of the interval $[0,1]$. For example, different genie aided channels are used in [6] for smaller values of $d$ than for large values of $d$ and, while equation (18) is derived in [6] using a bound effective for large $d$, the bound for $C(0.65)$ used in Corollary 1 is derived from the numerical value of $C_{17}(d)$ which is not as effective for $d$ larger than 0.8 (see Table IV in [6], where bound $C_{4}$ therein is what we called $C_{17}(d)$, while bound $C_{2}^{*}$ is used to deduce (18)). Thus, in order to obtain improved upper bounds for $\lim _{d \rightarrow 1} \frac{C(d)}{(1-d)}$ one effective approach would be to numerically evaluate $C_{n}(d)$ near $d=$ 0.65 for $n \geq 18$. This requires, however, high computational and spatial complexity and it is out of the scope of the present paper.

\section{ACKNOWLEGDMENTS}

The author would like to thank Dario Fertonani for useful discussions and for providing numerical data obtained during the preparation of [6].

\section{REFERENCES}

[1] R. L. Dobrushin, "Shannon's theorems for channels with synchronization errors," Problems of Information Transmission, vol. 3, no. 4, pp. 11-26, 1967.

[2] S. Diggavi and M. Grossglauser, "On information transmission over a finite buffer channel," IEEE Trans. Inform. Theory, vol. 52, no. 3, pp. 1226-1237, 2006.

[3] M. Drinea and M. Mitzenmacher, "Improved lower bounds for the capacity of i.i.d. deletion and duplication channels," IEEE Trans. on Inform. Theory, vol. 53, no. 8, pp. 2693-2714, 2007.

[4] Y. Kanoria and A. Montanari, "On the deletion channel with small deletion probability," submitted.

[5] A. Kalai, M. Mitzenmacher, and M. Suda, "Tight asymptotic bounds for the deletion channel with small deletion probabilities," in Proc. IEEE Intern. Symp. on Inform. Theory, 2010.

[6] D. Fertonani and T. M. Duman, "Novel bounds on the capacity of the binary deletion channel," IEEE Trans. Inform. Theory, vol. 56, no. 6, pp. 2753-2765, June 2010.

[7] M. Drinea and M. Mitzenmacher, "A simple lower bound for the capacity of the deletion channel," IEEE Trans. on Inform. Theory, vol. 52, no. 10, pp. 4657-4660, 2006.

[8] G. Pólya and G. Szegö, Problems and Theorems in Analysis, vol. 1, Springer-Verlag, 1976. 